\title{
Component and Parameterized Power Model for Cloud Radio Access Network
}

\author{
R.S.Alhumaima, M.Khan, H.S. Al-Raweshidy \\ WNCC, School of Engineering, Design and physical sciences, Brunel University London, \\ Uxbridge, Middx., UB8 3PH, UK \\ E-mail: \{Raad.Alhumaima,Muhammad.Khan,Hamed.Al-Raweshidy\}@brunel.ac.uk
}

\begin{abstract}
The 5G cellular network performance is measured by its spectral and energy efficiency (EE). Densifying the network might provide a solution. However this will lead to tremendous network infrastructure and increased Power Consumption (PC). Cloud Radio Access Network (CRAN) has emerged as a solutions to curtail the $\mathrm{PC}$ and deliver spectrum sharing in a cost-effective and energy efficient way.

Since the PC is an important key success for the upcoming generations, and in order to study and evaluate the power reductions achievable by C-RAN, a reliable Power Model (PM) is required. This paper proposes a component, linear and parameterised PMs to explore the individual components relevant for PC analysis, particularly for C-RAN architecture. The models quantify the EE by capturing the power consumed by individual components, and the amount of power reduced in the network. The model shows a dramatic reduction in the cooling $\mathrm{PC}(87.4 \%)$, whereas the total $\mathrm{PC}$ is reduced to about $(33.3 \%)$ compared to Macro Base Stations (BSs) deployment. Finally, accuracy comparison of the component and parameterized models has been presented.
\end{abstract}

\section{Introduction}

The increasing number of connected devices such as tablets, smart phones and laptops, coupled with an abundance of new types of bandwidth-hungry applications and services, has resulted in high data rate demands. This has led to an exponential increase in mobile data traffic in wireless and mobile communication networks [1]. According to Cisco and Ericson's recent forecast, mobile portable devices and connections will grow to 11.5 billion by 2019 , and thereon to more than a ten-fold increase in mobile data traffic between 2013 and 2018 [1]. Mobile operators are looking for innovative proposals, to help them meet this explosive demands for high bandwidth, high capacity, high security and faster connectivity. Consequently welcoming new designs, advanced digital signal processing (DSP) techniques and protocols [2]. However most of the proposals tend to rely on over-provisioning of resources, ensuring that the desired demands are met, such as spatial densification and spectral aggregation [3]. This leads to high PC and thus, high costs for network operators.

A novel architecture such as C-RAN has been suggested by both operators (e.g., NTT, KT, France Telecom/Orange. Telefonica, SoftBank/Sprint, and China Mobile), as well as equipment vendors (e.g., Alcatel-Lucent LightRadio, Nokia-Siemens Liquid Radio). C-RAN introduces cloud-computing concepts 
in cellular networks, by pooling the Base Band processing Units (BBUs) in a shared and centralised data processing centre [4], known as BBU pool or BBU cloud.

C-RAN has been emerged as standalone network architecture; it has been raised by the aforementioned network operators and vendors, as a solution for the next generation (5G) networks. CRAN also might benefit the existing Macro BS networks deployment, to yield Heterogeneous based Cloud Radio Access Networks (H-CRAN), in which, the deployed Macro BS cooperates and coexists with the proposed C-RAN scenario [5]. By doing so, the low-PC RRHs are deployed and cooperated with each other in the BBU pool to gain both high cooperation and PC reduction, whilst the Macro BSs are interfaced with the BBU pool, by the means of S1 and X2 protocols, for the data and control plane respectively, to coordinate the inter-tier interference between the Macro BS and the RRHs.

As for C-RAN, the main baseband physical procedures and processing of upper layers are executed in the BBU pool. Whilst the simple Radio Frequency (RF) front and symbol processing functions are tackled by the Remote Radio Head (RRH). In contrast to the eNodeB, where the entire communication layers processing has been implemented within the eNodeB [6]. Therefore, C-RAN is recognised to truncate the Capital Expenditures (CAPEX) and Operational Expenditures (OPEX), providing higher bit rates and higher EE. The RRHs are connected to the BBU pool via optical transport network and act like relays between the User Equipment (UE) and the BBU pool [5], [7]. The RRHs are densely deployed in the network with minimum cost, which distinguishes C-RAN from traditional systems, and present major functionalities of C-RAN.

Potential benefits of such architecture include: (i) Using advanced signal processing and coordination techniques to process signals through any neighbouring BBU(s) in the cloud. (ii) Allowing cognitive radio to enhance the efficiency of the network spectrum-utilization [8]. (iii) Reduction in OPEX of the network operators, due to fewer site visits, easy upgrading and maintenance and lower site lease. (iv) Ability to exploit processing load variations across BSs, by pooling the BSs in to fewer computing resources. This can allow the operators to reduce the PC by turning off the unwanted processors [9]. (v) Separation of the control and data plane for more controlled, intelligent and smart self-organised networks [10]. (vi) Cooling PC reduction, therefore; reduction in the total PC [11].

In the literature [12], [13], [14], and [15], the PC of different LTE BSs is described. Recent PC surveys of mobile networks, including mobile devices, BSs and core networks, shows that $80 \%$ of the PC results from the BS site.

A well embraced EE framework (E3F), EARTH project [13], is introduced to evaluate the PC by each component within the BS, as shown in Fig. 1, and therefore, the total BS and overall network PC. 


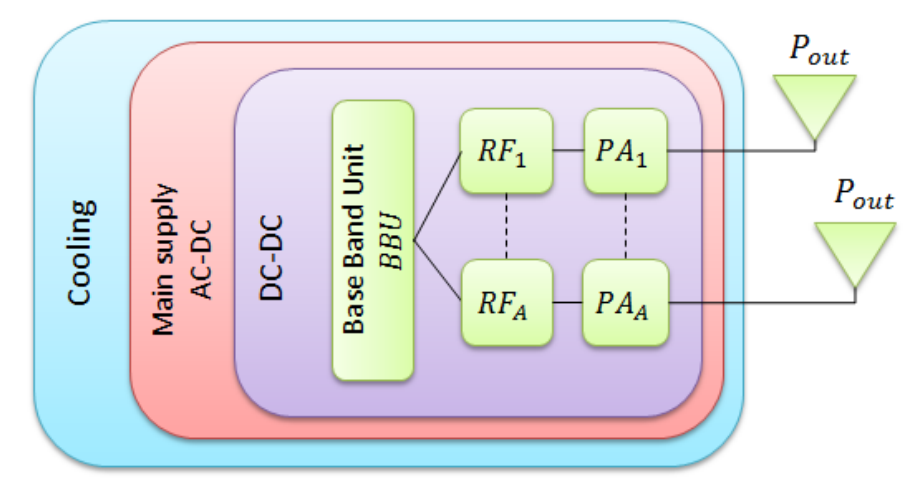

Fig.1. Base station transceiver block diagram.

Fig. 1 shows the breakdown structure of the simplified complete traditional BS, which can be generalised to all BS types i.e. Macro, Micro, Pico and Femto BSs. The PM in [12] identifies the fundamental BS site's blocks for energy evaluation. The main objectives of the model are:

- Mapping RF radiated output power at the antenna to the total BS site supply power, and the component and system level interfaces.

- Load fluctuations description over the day, considering long and short term traffic model.

- Large-scale model's deployment that covers small-scale scenarios and can be extended to larger geographical areas.

\subsection{Related Work}

Various researches have been focused on C-RAN throughput/capacity enhancing based optimization problems, as well as resource sharing and allocation amongst the BBUs. Without a certain attention given to answer the question: what/how C-RAN consume/reduce the PC? As the deployment/setup of any new communication technology/architecture/innovative design is costly, the pre-evaluation of the PC and power budget shaping is urgently required. To measure the PC of each part of the network, several proposals are presented, to describe either the PC of a certain component within the BS, or the PC of all the components in the BS on the system level. In [15], the radio frequency (RF), subsystem PC model emulation is investigated, the PM computes the RF's PC by considering the parameters: transmitted power, carrier frequency and bandwidth, as input to the PM.

A mathematical model in [16] is proposed to calculates the BS's total PC on components level, and based on the cost obtained by the model, the BS's PA might be turned off or switched to an idle state to reduce the $\mathrm{PC}$. 
On system level PMs, several proposals have been presented, the BS's PC in the early phases design is established in [17], that is end-to-end power estimation in heterogeneous LTE cellular systems, in which, a time and PM is configured, to ease fast PC assessment of different modem architectures. In [18] and [14], different State of the Art (SotA) LTE BSs' PM is investigated, and basic/simplified PM is presented relying on the data sheet of the BSs' components. In [19] and [12], complex LTE BS's PM is presented, providing estimation of the PC of different types of 3GPP LTE BSs, the presented models sweep through the functions complexity of each subcomponent to evaluate the total PC. These models are described as 'complex PM' by the EARTH project PMs in [20] and [13], which is used to parameterise and simplify the BS's PM of [12]. The parameterised PM of [14] is compared to [13], in terms of the number of radio chains and transmission bandwidth. EARTH PM is used to draw and shape the SotA BSs PC; when the PC of a BS can be linearized, and to exhibit a general platform to measure the EE.

With regards to resources allocation optimization in C-RAN and PC reduction techniques, a traffic based dynamic resource allocation algorithm has been proposed in [21], it allocates the minimum required centralised BBUs to serve all the tagged RRHs, by doing so, the PC has been reduced to $70 \%$ than required. In [22], a joint downlink and uplink power minimization through multi-user/multi-RRH association is proposed. Based on advanced beamforming design, interference coordination to reduce the PC is presented in C-RAN. While the resource sharing in H-CRAN is investigated, at three levels (spectrum, infrastructure, and network), in which the resource sharing key enabling technologies (i.e. Software Defined Networks (SDN), virtualization, and network function virtualization) are highlighted, and their effectiveness to H-CRAN is evaluated in [23].

It is to be noticed that, neither the aforementioned components level PC models, nor the system level PMs are able to address the PC of the upcoming hybrid networks, such as C-RAN based heterogeneous networks, SDN based heterogeneous C-RAN, and the standalone C-RAN deployment.

To the best of our knowledge, an advanced PC model for a components or system level of the CRAN, that answer the upfront question, has not been addressed in previous work, particularly, the BBU pool/RRH combination. In section 2, the system model of CRAN, the proposed component PM and its correspondent PC components is introduced. The total PC of the network is described in section 3. Section 4 introduces the parameterised PM. Section 5 provides the simulation results to verify the effectiveness of the proposed CRAN architecture. Finally, we conclude the paper in section 6. 


\subsection{Main Contributions}

The absence of a PM to consider the aforementioned considerations and to describe the EE for the hybrid, innovative and next generation networks shapes the presentation of this paper. The contributions have been summarized as follows:

1- Components PM is proposed to evaluate the PC of each part (component) of the C-RAN system. As deploying new network paradigm is mostly expensive, this work allows the network operators and vendors to measure the gain of C-RAN implementation over the existing deployment prior implementing the C-RAN architecture. Subsequently, the PC is calculated by combining the PC parts.

2- By wrapping the PM complexity of each component in the components model to a single value. This results a simplified parameterized model in which, the complex representation of the network PC has been extensively reduced. Since only few parameters vary constantly in the model, and most of the parameters remain static, the parametrised model can be utilized to evaluate the futuristic power saving approaches in C-RAN. Such as the effects of the number of antennas on total PC, transmission power reduction effects, antennas' deactivation, bandwidth adaptions and $\mathrm{PC}$ reduction by activating sleep modes.

3- Providing a comparison of C-RAN and Macro BS cooling and total PC. Different network characteristics regarding the BBU pool, Macro BS and RRHs, such as the transmitted power, complexity of functions, cooling and the optical network have been considerations. The models also evaluate the PC for varying network parameters such as antenna and bandwidth sweep. This shape a long-term/large-scale PM for holistic EE evaluation and analysis.

4- The accuracy comparison of both models, showing to which degree the parameterized model can meet components PM, has been expressed.

\section{Components PM}

The two main parts involved within the PM are:

- BBU pool, which contains several BBUs, each capable to serve one or many RRHs.

- $\quad$ RRH, with MIMO system supporting.

Both BBU pool and RRH are divided into sub components, which contribute to the PC model. Each component has its own power budget calculation. Fig. 2 shows the block diagram and the PC components of the C-RAN. 


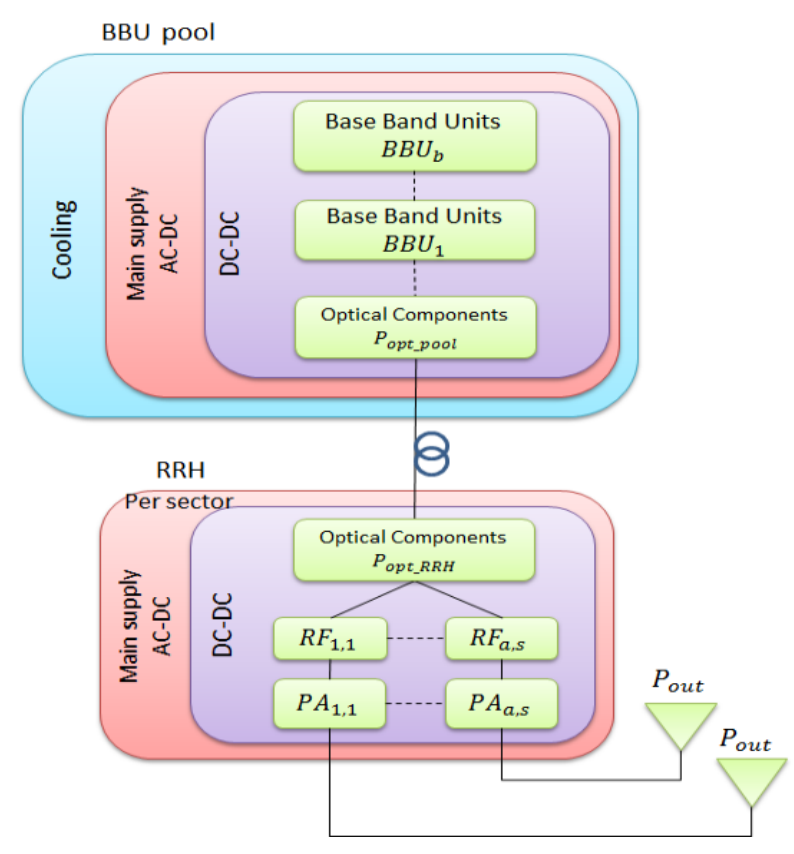

Fig.2. Block diagram of $C$-RAN transceiver.

\subsection{Base Band Unit Pool (BBU pool)}

The BBU pool is a consolidation of multiple BBUs in a form of a cloud or cluster. All the RRHs connected to the BBU pool have unified baseband processing and resource pools. The PC model for the BBU pool is considered to be the sum of the active BBUs in the BBU pool and other important components which are briefly discussed as follows;

2.1.1 Base Band Unit $(B B U)$ : The BBU is responsible for digital signal processing and generation before passing it to the RF transceiver. The digital computation and processing of the BBU can be measured in Giga Operation per Second (GOPS) and translated into power figures. This can be achieved by multiplication of the GOPS with the technology scaling factor revealing the operations performed per second per Watt [12]. A set of different BBU functions ( $\left.I_{B B}\right)$ such as the time and frequency domain processing, Forward Error Correction (FEC), Central Processing Units (CPU) and processing related to Common Public Radio Interface (CPRI) can be associated with GOPS. 40 GOPS/W is estimated to be the power cost of a large BBU [12]. The PM of the BBU can be expressed as

$$
P_{B B U}=\sum_{i \in I_{B B}} P_{i, B B U}^{r e f} A^{x_{i}^{A}} W^{x_{i}^{\beta}}
$$

Where $\mathrm{P}_{\mathrm{BBU}}$ denotes the $\mathrm{PC}$ of the $\mathrm{BBU}, \mathrm{P}_{\mathrm{i}, \mathrm{BB}}^{\mathrm{ref}}$, is the BBU's sub component $\mathrm{PC}$, i.e. $\mathrm{BBU}$ functions. $\mathrm{A}$ is the total number of Antenna/ Radio chains/ RF transceiver, $x_{i}^{A}$ is the scaling exponent of the number of radio chains, $\mathrm{W}$ is the share of the available bandwidth used in the transmission, $\mathrm{x}_{\mathrm{i}}^{\mathrm{W}}$ is the scaling exponent of the used bandwidth share. 
2.1.2 DC-DC Conversion (DC,P): The various components of the model require proper DC voltages for operation; therefore DC-DC convertors are placed wherever needed. DC-DC convertors have efficiency less than 1, thus it can be expressed as losses. It is mentioned in [13], [20], that the losses incurred by the overhead (i.e. power conversions and active cooling) scale linearly with the PC of other components which require such DC conversion, i.e. (BBUs, Optical devices, etc.). Therefore; the PC caused by DC conversion $\left(\boldsymbol{P}_{\boldsymbol{D C}, \boldsymbol{P}}\right)$ inside the BBU pool is given as:

$$
P_{D C, P}=\sum_{b=1}^{B} \sum_{r=1}^{R} \ell_{D C, P}\left(\eta_{D C, P}\right)\left(P_{B B U_{b}}^{r}+P_{o p t, P_{b, r}}\right)
$$

Where, $b \in\{1, \ldots, B\}$, denotes the number of active BBUs in the BBU pool, $r \in\{1, \ldots, R\}$, denotes the number of active RRHs, $P_{B B U_{b}}^{r}$ is the PC of $b$-th BBU, which is connected to $r$-th RRH, $P_{o p, P}$ is the PC by the optical device in the BBU pool which connects the b-th BBU to $r$-th RRH, $\ell_{D C, P}$ is the loss caused by DC-DC conversion; as a function of DC conversion efficiency $\left(\eta_{D C, P}\right)$, the loss function can be expressed as a decay function with an exponential decay constant $(x)$, this constant might differ amongst the devices when subjected to the manufacturer design quality, which eventually affects the value of the loss function. However, the losses can be expressed by

$$
\ell(\eta)=\ell_{o} e^{-\eta x}
$$

where $\ell_{\mathrm{o}}$ is the initial value of the losses at time $(t=0)$, and $\eta$ is the efficiency of the device.

The efficiency can be modeled as the ratio of maximum DC conversion output power $\left(P_{D C, P, \text { out } \text { max }}\right)$ to actual PC by the other DC power components

$$
\eta_{D C_{\text {pool }}}=\frac{P_{D C, P, \text { out }, \text { max }}}{\sum_{b=1}^{B} \sum_{r=1}^{R} P_{B B U_{b}}+P_{o p t, P} b, r}
$$

2.1.3 Main Supply, AC-DC-Conversion ( $\boldsymbol{M S}, \boldsymbol{P}$ ): The power form of the main supply grid has to be converted from AC to DC. This is done by the mains supply unit. The architecture of AC-DC convertors varies across vendors. The PC of this unit $\left(\boldsymbol{P}_{\boldsymbol{M S}, \boldsymbol{P}}\right)$ is generally modeled the same way as the DC-DC power conversion, and is given as:

$$
P_{M S, P}=\ell_{M S, P}\left(\eta_{M S, P}\right)\left(P_{D C, P}+\sum_{b=1}^{B} \sum_{r=1}^{R} P_{B B U_{b}}^{r}+P_{o p t, P}{ }_{b, r}\right)
$$

$\ell_{M S, P}$ denotes the measured losses of the MS power conversion as a function of AC-DC conversion efficiency $\left(\eta_{M S, P}\right)$. The MS's efficiency is the ratio of the maximum AC convertor output power $\left(P_{M S, P, \text { out }, \text { max }}\right)$, to the actual PC by the other components.

$$
\eta_{M S, P}=\frac{P_{M S, P, \text { out }, \text { max }}}{P_{D C, P}+\sum_{b=1}^{B} \sum_{r=1}^{R} P_{B B U_{b}}+P_{o p t, P}, r, r}
$$


2.1.4 Cooling: Cooling is responsible for the greatest energy waste [24]. BSs require active cooling and a large portion of the energy is wasted into BSs cooling. Cooling requirement may change depending on the geographical location, the position of BSs and the size of the cooling cabinet they are housed in. Cooling PC can be modeled as a fixed power loss, as it is a very slow operation, compared to the fast timing variations of the BBUs. The cooling unit is responsible for cooling each of the BBU pool's components, i.e. the BBUs, DC-DC, AC-DC conversion and the optical transceivers. Due to hosting several BBUs in the $\mathrm{BBU}$ pool; it requires more cooling however; cooling $\mathrm{PC}$ in the $\mathrm{BBU}$ pool with $\mathrm{B}$ number of BBUs, is much lower than the amount of PC by B number of Macro BSs. Cooling PC is modeled to be proportional to the PC of all other components in the BBU pool. If $\boldsymbol{\ell}_{\boldsymbol{c o o l}}$ is the cooling loss, then the cooling PC by the BBU pool $\left(\boldsymbol{P}_{\text {cool }}\right)$ can be calculated as

$$
P_{\text {cool }}=\ell_{\text {cool }}\left(P_{M S, P}+P_{D C, P}+\sum_{b=1}^{B} \sum_{r=1}^{R}\left(P_{B B U_{b}}^{r}+P_{o p t, P}, r\right)\right.
$$

\subsection{Remote Radio Head (RRH)}

The RRH consists of components with much lower operational complexity. It is equipped with a radio receiver chains (RFs) and power amplifiers (PAs) that scales linearly with the number of antenna used. RRH also consists of necessary voltage suppliers. The RRH PM can be broken down to the following sub sections:

2.2.1 Antenna (A): The antenna itself does not influence the PM, as their purpose is only to transmit/receive the signals to/ from end users. The number of antennas used in the system affects the PM, as each antenna requires RF and PA to provide the necessary required signal operations and amplification.

2.2.2 RF transceiver $(R F)$ : RF transceiver unit consists of an intermediate frequency and baseband interface. In-phase/quadrature signals (I/Q signals) and intermediate frequency signals are modulated/demodulated with baseband and intermediate frequency interface, respectively. RF unit is responsible for following functions:
a) Modulation/Demodulation the signals.
b) Voltage Controlled Oscillators (VCO) and Mixers.
c) Digital to analogue (DAC) and analogue to digital (ADC) convertors.
d) Low Noise Amplifiers (LNAs), gain amplifiers, Clocks.

2.2.3 Power Amplifier (PA): The PA is a prime element of consideration in the PM, as it consumes most of the power within the RRH. The PA amplifies the electrical signal received from the O/E convertor, before it is transmitted to the air interface by the antenna, and vice versa. PAs have low efficiencies at low antenna transmission power $\left(\boldsymbol{P}_{\text {out }}\right)$, however in rare cases its efficiency can reach up to $54 \%$ if high transmission powers are intended at the antenna(s) [19]. Due to the strong fluctuation in transmission powers of the Orthogonal Frequency Division Multiple Access (OFDM) signals, PA usually operates with low efficiencies. Modeling the PC of a PA requires the following important parameters to be considered:

a) Output transmitted power $\left(P_{\text {out }}\right)$ of the antenna.

b) Output power of the PA $\left(P_{T X}\right)$.

c) The share of maximum bandwidth $(W)$, that an antenna uses, i.e. the actual number of the Physical Resource Blocks $\left(N_{P R B}\right)$ that occupies a certain bandwidth for transmission; $P_{T X}=$ $P_{\text {out }} W$. 
The PA's PC $\left(P_{P A}\right)$ is affected by its efficiency $\left(\eta_{P A}\right)$, which is a function of $P_{T X}$. The PA's PC can be modeled as:

$$
P_{P A}=\frac{P_{T X}}{\eta_{P A}\left(P_{T X}\right)}
$$

2.2.4 RRH's Power Conversion: An AC-DC and DC-DC voltage converters are required to provide the necessary voltages supplies to the RRH's components such as the PA, RF and the optical components. The PC of the RRH's DC conversion $\left(\boldsymbol{P}_{\boldsymbol{D C}, \boldsymbol{R}}\right)$ is modeled by considering its losses $\left(\boldsymbol{\ell}_{\boldsymbol{D C}, \boldsymbol{R}}\right)$ as a function of the efficiency $\left(\boldsymbol{\eta}_{\boldsymbol{D} C, \boldsymbol{R}}\right)$ along with the power requirement of all other components;

$$
P_{D C, R}=\ell_{D C, R}\left(\eta_{D C, R}\right)\left(P_{o p t, R}+\sum_{b=1}^{B} \sum_{r=1}^{R} \sum_{a=1}^{A}\left(P_{P A}+P_{R F}\right)_{a}^{r, b}\right)
$$

$a \in\{1, \ldots, A\}$ denotes the antenna/RF chains number, $r \in\{1, \ldots, R\}$, denotes the number of RRHs. $P_{R F a}^{r, b}, P_{P A_{a}}^{r, b}$ denote the PC of the $a$-th RF and PA respectively, within $r$-th RRH which is connected to $b$ th BBU, $P_{o p t, R}$ is the PC of the optical device inside the RRH. $\eta_{D C, R}$ is the ratio of the maximum output power of the DC convertor $\left(P_{D C, R, o u t, \max }\right)$, to the actual PC by other components

$$
\eta_{D C, R}=\frac{P_{D C_{-R R H, o u t, \max }}}{P_{o p t, R}+\sum_{a=1}^{A}\left(P_{R F}+P_{P A}\right)_{a}}
$$

The MS/AC-DC convertor for RRH can be modeled in the same fashion as the BBU pool's MS, therefore its PM is configured

$P_{M S, R}=\ell_{M S, R}\left(\eta_{M S, R}\right)\left(P_{D C, R}+P_{o p t, R}+\sum_{b=1}^{B} \sum_{r=1}^{R} \sum_{a=1}^{A}\left(P_{P A}+P_{R F}\right)_{a}^{r, b}\right)$

where $\ell_{\mathrm{MS}, \mathrm{R}}$, denotes the MS losses as a function of the conversion efficiency $\left(\eta_{M S, R}\right)$, which is modelled

$$
\eta_{M S, R}=\frac{P_{M S, R, \text { out }, \text { max }}}{P_{D C, R}++P_{o p t, R}+\sum_{a=1}^{A}\left(P_{R F}+P_{P A}\right)_{a}}
$$

$\mathrm{P}_{\mathrm{MS}, \mathrm{R}, \text { out } \text { max }}$ denotes the maximum output power of the MS convertor.

2.2.5 RRH Cooling: Nokia Siemens Networks (NSN), states that BSs with total PC of $500 \mathrm{~W}$ or less (except the output power of the BS $\left.\left(\boldsymbol{P}_{\text {out }}\right)\right)$, do not require cooling system [16]. This is applicable for the RRH, which is composed of components i.e. (PA, RF and Optical components) having overall power less than $500 \mathrm{~W}$. Therefore the only overhead for RRH is the supply power, and its cooling PC is negligible [13].

\subsection{Optical Transceiver PC}

The access line that connects the multiple RRHs to the BBU pool is often known as the "front-haul". Optical fiber is the most promising infrastructure in the front-haul due to its large capacity support and scalability. The optical transceiver is responsible for the conversion of the signals from electrical to optical 
light with a certain wavelength $(\lambda)$ and vice versa. Several factors influence the optical transceiver operation such as the technology used, the required output power and the operating condition [25]. These in turn affect the PM.

Optical transceivers can be divided into two modules from the PC perspective i.e. Optical Transmitter Module (OTM) in which, OFDM electrical signals are modulated over optical carrier using an external or direct modulated laser. While the optical OFDM signals are detected by the Optical Receiver Module (ORM) either by Direct Detection (DD) or Coherent Detection (CO-D). The optical transceiver PC described in [25] is given as;

$$
P_{\text {transceiver }}=\left(P_{\text {laser }}+P_{\text {driver }}+P_{I / O}\right)_{T X}+\left(P_{P D}+P_{\text {amp }}+P_{I / O}\right)_{R X}
$$

Where $P_{\text {laser }}, P_{\text {driver }}, P_{P D}, P_{a m p}$, and $P_{I / O}$ are the PC by the direct-modulated laser, laser driving electronics, photo detector, trans-impedance amplifier (TIA) and the limiting amplifier (LA), and the electrical I/O interface, respectively. Typical PCs of commercial point-to-point (PtP) and point-tomultipoint (PtMP) optical transceivers are $1 \mathrm{~W}$ and $1.5 \mathrm{~W}$, respectively. The model uses PtP transceivers, as this type does not have passive optical power splitter and offers relaxed link budget, i.e. PtP link loss is governed by only the distance and the used operating wavelength. In contrast to the PtMP in which; constrained link budget about (20-35) dB is required; due to wavelength sharing nature in the same fiber. Whilst the link loss of a PtP is as low as $6 \mathrm{~dB}$ with $20 \mathrm{~km}$ network reach [26].

\section{Total PC}

The total PC $\left(P_{C R A N}\right)$ of the CRAN network; is the sum of the BBU pool and RRHs PC, it is formulated

$$
P_{C R A N}=P_{\text {Pool }}+P_{R R H}
$$

BBU pool $\left(P_{P o o l}\right)$ is calculated by aggregating the $\mathrm{PC}$ of all active components, and calculated as:

$$
P_{\text {Pool }}=P_{C o o l}+P_{M S, P}+P_{D C, P}+\sum_{b=1}^{B} \sum_{r=1}^{R} \sum_{a=1}^{A} P_{B B U_{b, r, a}}+P_{o p t, P_{b, r}}
$$

$P_{B B U_{b, r, a}}$, is the PC by $b$-th BBU; attached to $r$-th RRH; which mounts $a$-th antenna. RRHs can be served by any active BBU within the BBU pool. This means that BBU-RRH mapping can be dynamic depending on the traffic conditions [27]. This service diversity is considered in the model. The total PC by RRHs is formulated 


$$
P_{R R H}=P_{M S, R}+P_{D C, R}+\sum_{b=1}^{B} \sum_{r=1}^{R} \sum_{a=1}^{A}\left(P_{P A}+P_{R F}\right)_{r, a}^{b}+P_{o p t, R}
$$

$P_{P A r, a}^{b}, P_{R F r, a}^{b}$ denotes the PC by $a$-th antenna's PA and RF respectively; within $r$-th RRH; which is attached to $b$-th BBU.

\section{Parameterised and Linear PM}

The PC of a BS can be expressed by a load-independent share $\left(P_{\text {static }}\right)$ and an added load-dependent dynamic share that increases linearly with the power slope $\left(\Delta_{\mathrm{P}}\right)$ as shown in Fig.3. The maximum supply power of a BS $\left(P_{\text {supply }}\right)$ depends on the maximum transmitted power $\left(P_{\max }\right)$, the share of bandwidth used and the number of radio chains/antennas. Maximum supply PC $\left(P_{1}\right)$ is reached when transmitting at maximum power $\left(P_{\max }\right)$.

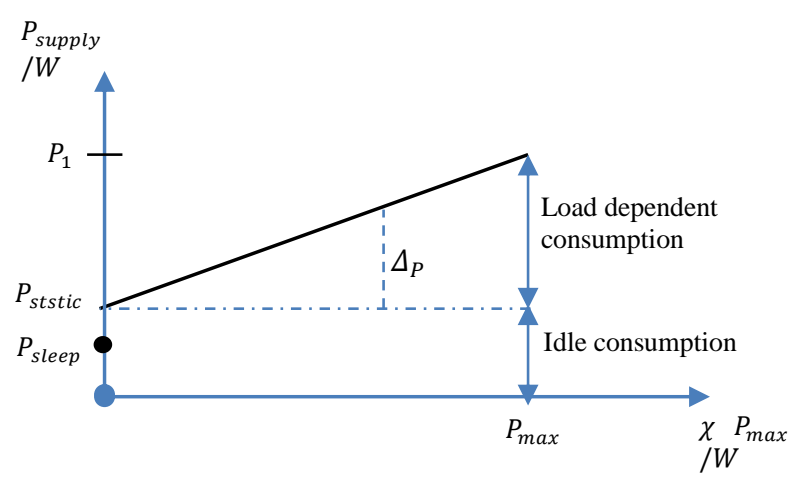

Fig.3. BS Load- dependent PM

Furthermore, when the BS is not transmitting, it may enter into sleep mode with lower PC $\left(P_{\text {sleep }}\right)$. The total power supply is formulated

$$
P_{\text {supply }}(\chi)= \begin{cases}(R)\left(P_{1}+\Delta_{p} P_{\max }(\chi-1)\right) & \text { if } 0<\chi \leq 1 \\ (R) P_{\text {sleep }} & \text { if } \chi=0\end{cases}
$$

Where $P_{1}=P_{\text {static }}+\Delta_{p} P_{\text {max }}, \chi$ is the scaling parameter of the load share, i.e. if $\chi=1$, the system is fully loaded, e.g. transmitting at full bandwidth and power, while the system is idle when $\chi=0$. To briefly understand how different parameters contribute on this model, the maximum supply PC $\left(P_{1}\right)$ is parameterised. The parameterised model encompasses the architectural details and highlights parameters which are either assumed to be constant, or having negligible effects such as lookup tables, GOPS, manufacturing details. To this extent, the following approximations are made further to the SotA BSs' PMs: 
- The BBU and RF PC, both scale linearly with the number of antennas (A) and the bandwidth $(W)$, i.e., $P_{B B U}=A \frac{W}{10 M H z}\left(P_{B B U}^{p m}\right)$ and $P_{R F}=A \frac{W}{10 \mathrm{MHz}}\left(P_{R F}^{p m}\right)$. Where $P_{B B U}^{p m}, P_{R F}^{p m}$ are the PC by the BBU and RF in the parameterized model, respectively.

- The PC of PA depends on the maximum power transmitted per antenna $\left(P_{\text {max }} / A\right)$ and its efficiency $\left(\eta_{P A}\right)$. The feeder loss between PA and the antenna can be ignored for the RRH [13]; i.e., $\sigma_{f e e d e r}=$ 0 since the PA is placed close to the antenna. The PA's PC can be represented as $P_{P A}=$ $P_{\text {max }} / A \eta_{P A}$.

The PA efficiency is varying at different transmission powers, and assumed to decrease by a factor of $\gamma$ for each halving of the transmitted power. Thus it is maximum (per single antenna), when the PA's maximum transmission power during operation $\left(P_{\max }\right)$ is equal to the maximum PA's data sheet transmission power $\left(P_{P A, l i m i t}\right)$, heuristically the efficiency is described by [14];

$$
\eta_{P A}=\eta_{P A, \max }\left[1-\gamma \log _{2}\left(P_{P A, l i m i t} / \frac{P_{\max }}{A}\right)\right]
$$

- DC-DC, AC-DC conversions as well as cooling PC, scale linearly with other components PC and are approximated by the loss factors $\sigma_{D C, P}, \sigma_{M S, P}, \sigma_{c o o l}, \sigma_{D C, R}$ and $\sigma_{M S, R}$ for BBU pool DC, BBU pool MS, cooling, RRH's DC, and RRH's MS loss factors respectively.

- The optical transceivers PC $P_{o p t, P}$ and $P_{o p t, R}$ scale linearly with the number of BBUs and RRHs in the network.

- The losses incurred by the optical fiber between BBU pool and the RRH is approximated by the loss factor $\left(\sigma_{\text {optical }}\right)$, it can be adapted to meet the fiber length and number of connectors and splices used.

The maximum PC $\left(P_{1}\right)$ can be formulated by aggregation of the PC of single BBU in the BBU pool $\left(P_{\text {pool }}^{p m}\right)$ serving a single RRH $\left(P_{R R H}^{p m}\right)$ and is given as:

$$
\begin{gathered}
P_{1}=P_{p o o l}^{p m}+P_{R R H}^{p m} \\
=\frac{A \frac{W}{10 M H z}\left(P_{B B U}^{p m}\right)+P_{o p t, P}}{\left(1-\sigma_{D C, P}\right)\left(1-\sigma_{M S, P}\right)\left(1-\sigma_{\text {cool }}\right)}+\frac{A \frac{W}{10 M H z}\left(P_{R F}^{p m}\right)+\frac{P_{\max }}{A \cdot \eta_{P A}}+P_{o p t, R}}{\left(1-\sigma_{D C, R}\right)\left(1-\sigma_{M S, R}\right)\left(1-\sigma_{o p t i c a l}\right)}
\end{gathered}
$$

Then the total number of $R$ RRHs and $B$ BBUs is considered to obtain the total PC of the network $\left(P_{\text {supply }}\right)$

$$
P_{\text {supply }}=B \cdot P_{\text {pool }}^{p m}+R \cdot P_{R R H}^{p m}
$$




\section{Results and Discussion}

The proposed parameterised model is used to approximate the PC, and compared with the parameterised PM in [14], which breaks down and measures the PC of the traditional BSs, in terms of bandwidth used, varying antenna numbers, varying Macro BS sectors, and varying RRHs. Parameters are chosen according to [13] and [14] when possible. The parameters have been adjusted in some cases aiming fair comparison, especially the RRH's transmitted power (Average $\mathrm{P}_{\max }$ ), i.e. it is assumed 20W in [13], while it is assumed 40W in this work to match the parameters settings of the compared work [14]. The resulting parameters are provided in Table I, which summarises the measured SotA PC of LTE Macro BS and RRH.

Table 1 Parameters Breakdown

\begin{tabular}{|c|c|c|c|c|}
\hline \multicolumn{2}{|c|}{ Component } & Unit & Macro BS & RRH \\
\hline \multicolumn{2}{|c|}{ Average $\mathrm{P}_{\max }$} & $\mathrm{W}$ & 40 & 40 \\
\hline $\mathrm{PA} /$ & Total $\mathrm{P}_{\mathrm{PA}}$ & W & 80 & 40 \\
\hline \multicolumn{2}{|c|}{ PA eff, $\eta_{\mathrm{PA}}$} & - & 0.36 & 0.31 \\
\hline \multirow[t]{12}{*}{ PA limi } & & W & 80 & 80 \\
\hline & $\mathrm{P}_{\mathrm{TX}}$ & $\mathrm{W}$ & 6.8 & 6.8 \\
\hline & $\mathrm{P}_{\mathrm{RX}}$ & $\mathrm{W}$ & 6.1 & 6.1 \\
\hline & $\mathrm{P}_{\mathrm{RF}}^{\mathrm{pm}}$ & $\mathrm{W}$ & 12.9 & 12.9 \\
\hline & $\mathrm{P}_{\mathrm{BBU}}^{\mathrm{pm}}$ & $\mathrm{W}$ & 29.4 & 29.4 \\
\hline & $\sigma_{\text {feeder }}$ & $\mathrm{dB}$ & -3 & 0 \\
\hline & $\mathrm{P}_{\mathrm{opt}, \mathrm{p}}$ & $\mathrm{W}$ & - & 1 \\
\hline & $P_{o p t, R}$ & W & - & 1 \\
\hline & $\sigma_{\mathrm{DC}}$ & - & 0.075 & 0.075 \\
\hline & $\sigma_{\mathrm{MS}}$ & - & 0.09 & 0.09 \\
\hline & $\sigma_{\text {cooling }}$ & - & 0.1 & 0 \\
\hline & $\mathrm{P}_{1}$ & $\mathrm{~W}$ & 460.4 & 133 \\
\hline \multicolumn{2}{|c|}{$\Delta_{\mathrm{P}} * 10 \mathrm{MHz}$} & - & 4.2 & 2.8 \\
\hline \multicolumn{2}{|c|}{$P_{\text {static, sleep }}$} & W & 324 & 77 \\
\hline \multicolumn{2}{|c|}{ \#Sectors } & $\#$ & 3 & 1 \\
\hline \multicolumn{2}{|c|}{ \#Antenna } & $\#$ & $1,2,4$ & $1,2,4$ \\
\hline
\end{tabular}

The PM is simple to adapt, and to approximate different vendors' configurations, by changing the individual parameters in order to observe the variation in the resulting PC. The model is verified for one, two, and four antennas. The number of RRHs and Macro BS's sectors are up to 60. This number can be increased according to vendors' architectural demands. Each Macro BS is considered to mount 3 sectors. 
The increased band width above $10 \mathrm{MHz}$ is expected to increase the RF and BBU's PC, while other parameters such as losses and transmission power are expected to remain unaffected by the system bandwidth. As the number of Macro BS's sectors increases, the cooling PC is reduced as shown in Fig. 4, compared to the increased number of BBUs in the BBU pool.

Fig. 4 shows the cooling PC of C-RAN power model compared to traditional Macro BS for one, two and four antenna configurations. It shows a dramatic decrease in the PC, due to the fact that the PA and RF components are no longer contributing to the cooling PC in the BBU pool, on the contrary of the Macro BS. The result shows that for any antenna configuration, cooling PC has been reduced to about $87.4 \%$. For example, when $20 \mathrm{BBU}$ are used (the vertical black arrow in Fig. 4), cooling PC reduction can be calculated by using percentage change rule, i.e. $\frac{(V 1-V 2)}{|V 1|} * 100 \%=\frac{(4000-500)}{|4000|} * 100 \% \approx 87.4 \%$. More reduction might be expected when increasing the number of BBUs.

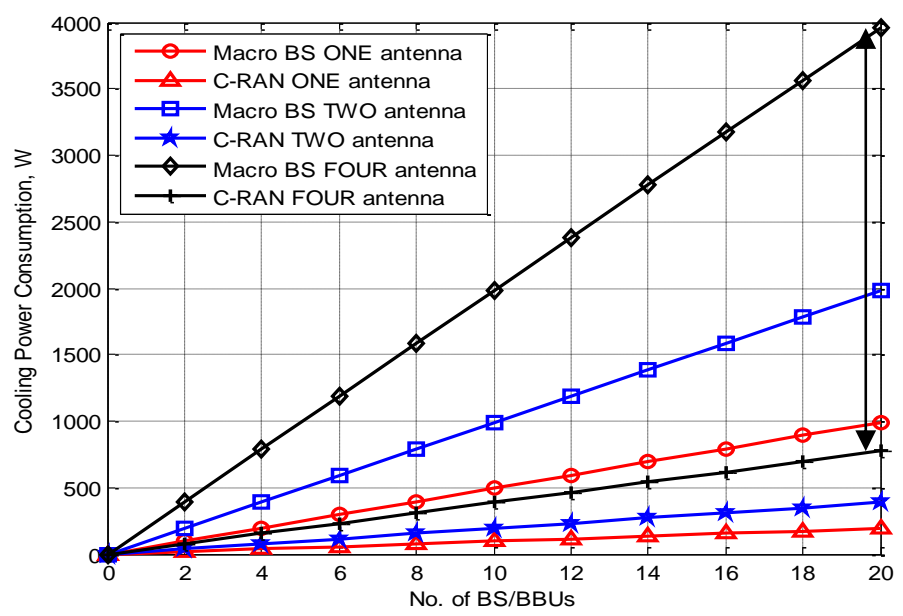

Fig. 4. Comparison of C-RAN cooling PC and PM of Macro BSs [14] with one, two and four antennas configurations.

While Fig. 5, shows the effect of reduced cooling PC on the total network PC, with varying numbers of sectors/RRHs. By using the percentage rule for the values circled in Fig.5, it was found that the percentage change reduction in the total $\mathrm{PC}$ is about $33.3 \%$.

For the sake of comparison, it is assumed that the number of sectors in Macro BS equal to the number of RRHs, i.e. each BBU is assumed to serve $1 \mathrm{RRH} /$ sectors, and each BS considered supporting 3 Sectors/RRHs. Despite the addition of optical components PC to the proposed PM, considerable lower total PC is achieved rather than in [14], as shown in Fig. 5. 


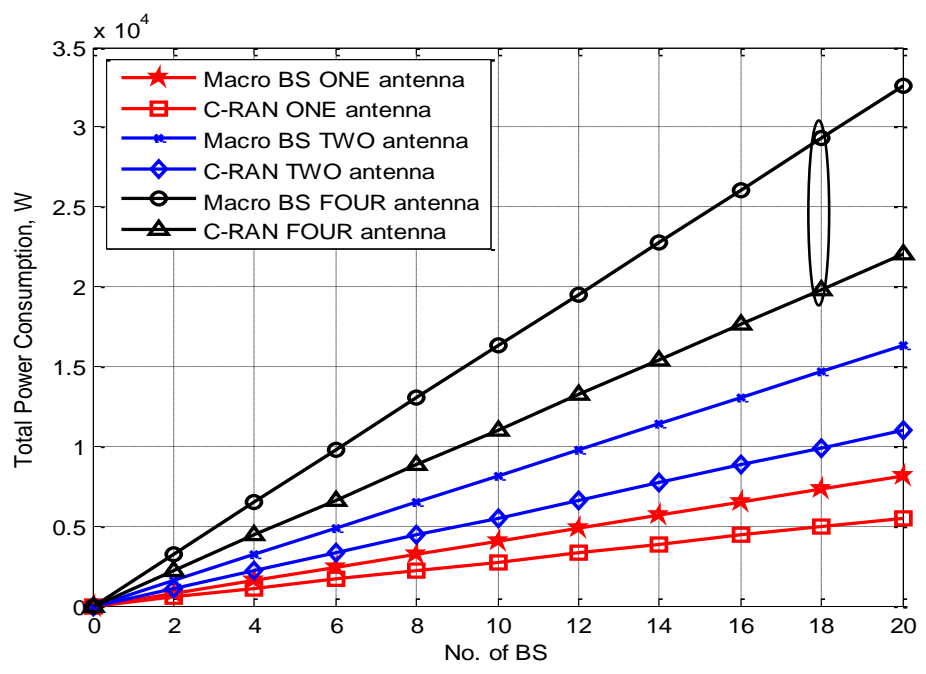

Fig. 5. Comparison of C-RAN total PC model with PM [14] of Macro BSs. 20 Macro BSs (each have 3 sectors) serving 60 Sectors/ RRHs and 60 BBUs serving 60 RRHs/sectors with one, two, and four antennas configurations.

The proposed model shows extra PC reduction compared to Fig. 5, considering the case of switching off some of the BBUs during off-peak hours. It is assumed that 60 RRHs attached to only 20 BBUs. The PC is shown in Fig. 6.
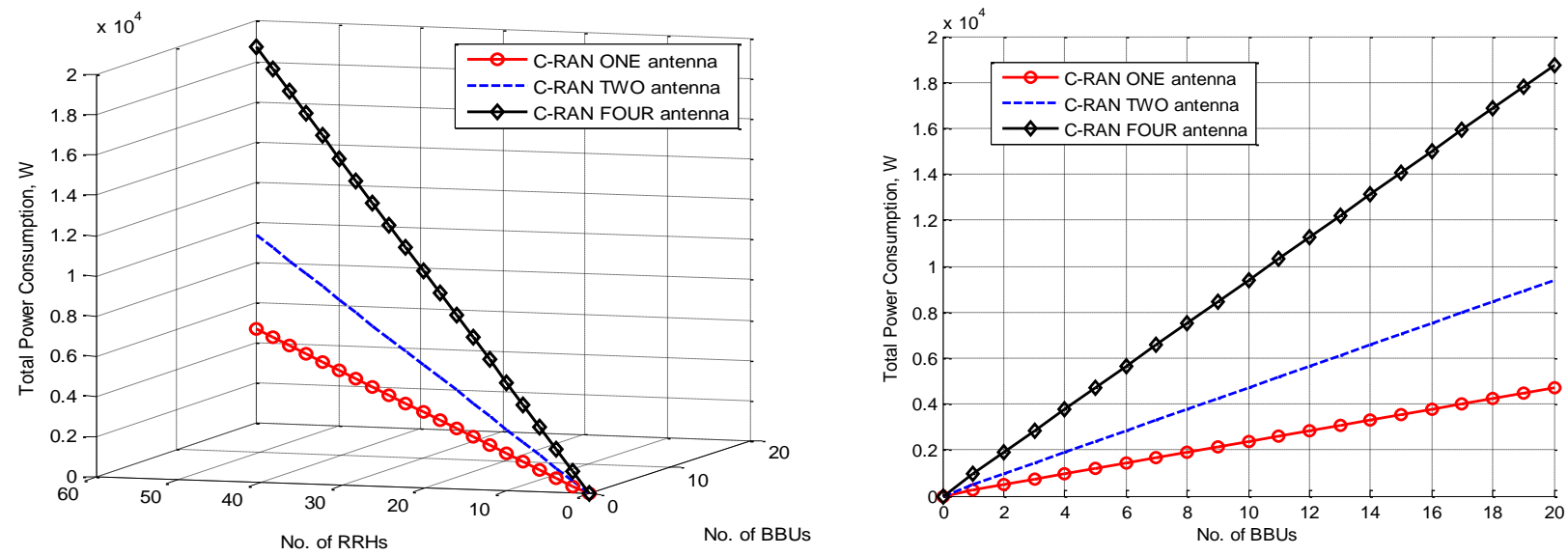

Fig. 6 left. 3 dimensional view of C-RAN total PC with varying number of BBUs i-e up to 20, and up to 60 RRHS, Having one, two and four antennas configurations. Fig 6 right. is the 2 dimensional view of Fig. 6 left.

Fig.4, Fig. 5, and Fig. 6, show a reduction in PC based on the parameterised model and compared to [14]. Fig.7 shows the total PC as a function of the system's bandwidth share and varying numbers of antennas. It is observed that with four transmitting antennas, the PC by C-RAN approaches the Macro BSs PC, only when it uses nearly the entire bandwidth share and much lower PC otherwise. 


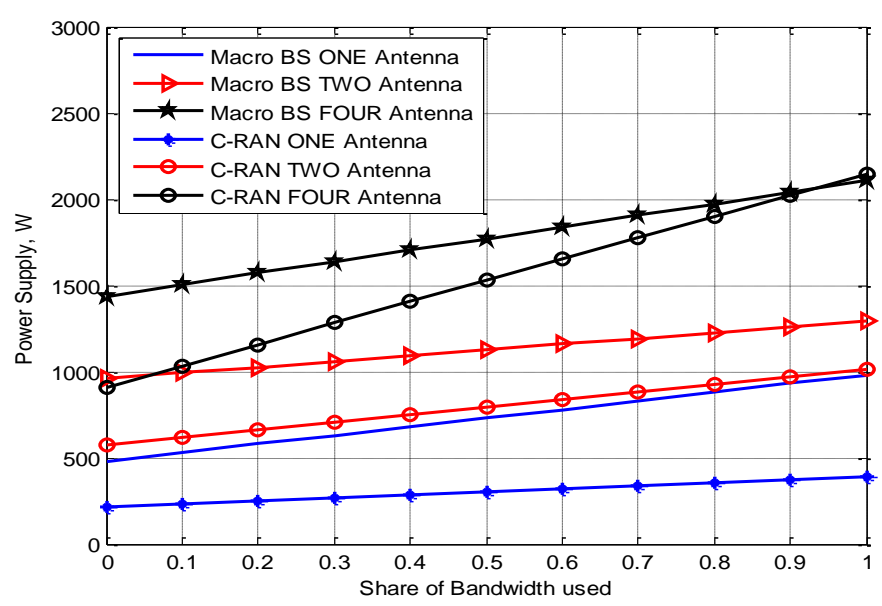

Fig. 7. Comparison of C-RAN total PC with [14], for $10 \mathrm{MHz}$ band width.

The accuracy of the simplified parameterized model and the components model can be evaluated using the decay function (3). The main key comparison can be done by knowing the initial losses $\ell_{0}$ of each sub components and the exponential decay constant $(x)$, which have been subjected to the manufacturer design. However, to compare both the components and the parameterized models, some assumption has been made to the component PM:

1- It is assumed that the efficiency $\eta$ is $90 \%$ for all the sub components (i.e. DC, AC convertors), Cooling loss $\left(\ell_{\text {cool }}\right)$ is 0.1 and the initial loss $\ell_{\mathrm{o}}$ is 0.009 .

2- Using (3) to evaluate the losses values which are required in (2),(5),(9), and (11).

3- Calculate the total components PM using (14).

4- Compare with the parameterized model of (19).

Based on the subcomponent datasheet, and for different values of a given exponential decay constant $(x)$, the results show as accurate parameterized as flexible component PM. Fig (8) shows the total PC of CRAN with different $x$ values and different number of RRHs, using one antenna configuration. 


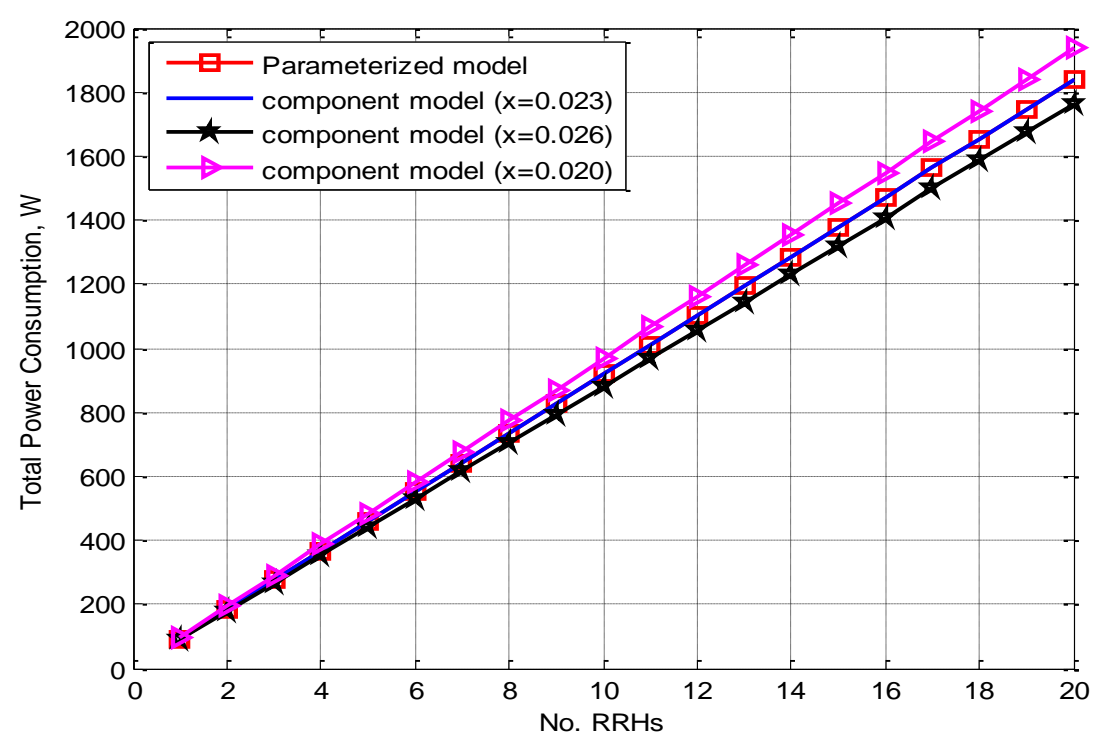

Fig. 8. Accuracy comparison of the total PC of the components and parameterized model with different number of (x) values and different number of RRHs, using only one antenna configuration.

\section{Conclusion and future work}

Together, components and a parameterised PM are provided in this paper, which allows the PC calculation of C-RAN according to operational parameters and varying vendor configurations. The model is applicable and simple, to meet the new generation communication systems requirements. A comparison of the model performance has been made with previous work, regarding cooling and total PC, with varying parameters such as antenna and bandwidth sweep.

The model has shown that the C-RAN network reduced the total PC to about $33.3 \%$ as it reduced the cooling PC to about $87.4 \%$ when compared to traditional MBS architecture. The results also show that the parameterized PM is as accurate as the component PM.

As for the future work, gathering the two parts i.e. C-RAN and Macro BS, has yielded H-CRAN as a promising architecture suggested, to enhance the EE of the upcoming generation. Using the proposed model to evaluate H-CRAN PC might give a wider vision, about the power figures of such extended architecture, when the Macro BS and C-RAN collaborate in the area of interest. 


\section{References}

1 Zhou, T., Huang, Y., Fan, L., Yang, L.: 'Load-aware user association with quality of service support in heterogeneous cellular networks' Commun. IET, 2015, 9, (4), pp. 494-500.

Sciancalepore, V., Mancuso, V., Banchs, A.: 'BASICS: Scheduling base stations to mitigate interferences in cellular networks', in 'World of Wireless, Mobile and Multimedia Networks (WoWMoM), IEEE 14th International Symposium and Workshops on a' , 2013, pp. 1-9.

Bhushan, N., Li, J., Malladi, D., et al.: 'Network densification: the dominant theme for wireless evolution into 5G'Commun. Mag. IEEE, 2014, 52, (2), pp. 82-89.

Xiong, N., Han, W., Vandenberg, A.: 'Green cloud computing schemes based on networks: a survey’ Commun. IET, 2012, 6, (18), pp. 3294-3300.

Peng, M., Zhang, K., Jiang, J., Wang, J., Wang, W.: 'Energy-Efficient Resource Assignment and Power Allocation in Heterogeneous Cloud Radio Access Networks'Veh. Technol. IEEE Trans., 2014, (99), pp. 1.

Sundaresan, K., Arslan, M.Y., Singh, S., Rangarajan, S., Krishnamurthy, S. V: 'FluidNet: A Flexible Cloudbased Radio Access Network for Small Cells', in 'Proceedings of the 19th Annual International Conference on Mobile Computing and Networking', ACM, 2013, pp. 99-110.

Liu, C., Zhang, L., Zhu, M., Wang, J., Cheng, L., Chang, G.-K.: 'A Novel Multi-Service Small-Cell Cloud Radio Access Network for Mobile Backhaul and Computing Based on Radio-Over-Fiber Technologies'Light. Technol. J., 2013, 31, (17), pp. 2869-2875.

Ghamari Adian, M., Aghaeinia, H., Norouzi, Y.: 'Spectrum sharing and power allocation in multi-inputmulti-output multi-band underlay cognitive radio networks' Commun. IET, 2013, 7, (11), pp. 1140-1150.

9 Bhaumik, S., Chandrabose, S.P., Jataprolu, M.K., et al.: 'CloudIQ: A Framework for Processing Base Stations in a Data Center', in 'Proceedings of the 18th Annual International Conference on Mobile Computing and Networking', ACM, 2012, pp. 125-136.

10 Dirani, M., Altman, Z.: 'Self-organizing Networks in Next Generation Radio Access Networks: Application to Fractional Power Control'Comput. Netw., 2011, 55, (2), pp. 431-438.

11 Fei, Z., Xing, C., Li, N., Zhu, D., Lei, M.: 'Leakage-based distributed minimum-mean-square error beamforming for relay-assisted cloud radio access networks' Commun. IET, 2014, 8, (11), pp. 1883-1891.

12 Desset, C., Debaillie, B., Giannini, V., et al.: 'Flexible power modeling of LTE base stations', in 'Wireless Communications and Networking Conference (WCNC), 2012, pp. 2858-2862

13 Auer, G., Giannini, V., Desset, C., et al.: 'How much energy is needed to run a wireless network?'Wirel. Commun. IEEE, 2011, 18, (5), pp. 40-49.

14 Holtkamp, H., Auer, G., Giannini, V., Haas, H.: 'A Parameterized Base Station Power Model' Commun. Lett. IEEE, 2013, 17, (11), pp. 2033-2035.

15 Musiige, D., Vincent, L., Anton, F., Mioc, D.: 'LTE RF subsystem power consumption modeling', in 'Consumer Electronics (GCCE), 2012 IEEE 1st Global Conference on', 2012, pp. 645-649. 
16 Chatzipapas, A., Alouf, S., Mancuso, V.: 'On the minimization of power consumption in base stations using on/off power amplifiers', in 'Online Conference on Green Communications (GreenCom), 2011, pp. 18-23.

17 Wang, B., Xu, Y., Hasholzner, R., Rosales, R., Glass, M., Teich, J.: 'End-to-end power estimation for heterogeneous cellular LTE SoCs in early design phases', in 'Power and Timing Modeling, Optimization and Simulation (PATMOS), 24th International Workshop on' , 2014, pp. 1-8. NETWORKS: OVERVIEW AND RECOM- MENDATIONS'Prog. Electromagn. Res., 2012, 132, pp. 255274.

19 Liu, K., He, J., Ding, J., Zhu, Y., Liu, Z.: 'Base station power model and application for energy efficient LTE', in 'Communication Technology (ICCT), 15th IEEE International Conference on', 2013, pp. 86-92.

20 Katranaras, M.I. and E.: 'Energy efficiency analysis of the reference systems, areas of improvements and target breakdown'public Deliv. D2.3 EARTH.

21 Khan, M., Alhumaima, R.S., Al-Raweshidy, H.S.: 'Reducing energy consumption by dynamic resource allocation in C-RAN', in 'Networks and Communications (EuCNC), European Conference on', 2015, pp. 169-174.

22 Luo, S., Zhang, R., Lim, T.J.: 'Downlink and Uplink Energy Minimization Through User Association and Beamforming in C-RAN'Wirel. Commun. IEEE Trans., 2015, 14, (1), pp. 494-508.

23 Marotta, M.A., Kaminski, N., Gomez-Miguelez, I., et al.: 'Resource sharing in heterogeneous cloud radio access networks'Wirel. Commun. IEEE, 2015, 22, (3), pp. 74-82.

24 R. Kumar, L.M.: 'Conceptualizing green IT and data center power and cooling issues', Gartner Inc, 2007. https://www.gartner.com/doc/519717/conceptualizing-green-it-data-center.

25 Tucker, R.S., Hinton, K.: 'Energy Consumption and Energy Density in Optical and Electronic Signal Processing'Photonics Journal, IEEE, 2011, 3, (5), pp. 821-833.

26 Lee, K.L., Sedighi, B., Tucker, R.S., Chow, H., Vetter, P.: 'Energy efficiency of optical transceivers in fiber access networks [invited]'Opt. Commun. Networking, IEEE/OSA J., 2012, 4, (9), pp. A59-A68.

27 Namba, S., Warabino, T., Kaneko, S.: 'BBU-RRH switching schemes for centralized RAN', in 'Communications and Networking in China (CHINACOM), 7th International ICST Conference on', 2012, pp. $762-766$. 